\title{
Multiple Elastic Retrograde Intramedullary Nailing for Adult Humeral Diaphyseal Fractures
}

\author{
Yogesh S Salphale*, D Ortho, MS, DSC, LLB and Wasudeo Gadegone, MS, MNAMS \\ Consultant Trauma Surgeon, Department of Orthopaedics, Shushrusha Multispecialty Hospital, India
}

\section{Introduction}

The humeral shaft fractures are amenable for conservative treatment as well as external fixation, open reduction and internal fixation, and anterograde or retrograde intramedullary (IM) nailing [1]. IM locked nails have provided excellent outcomes in terms of fracture biology and cosmetic appearance because of the relatively small incision involved. Concern about the iatrogenic rotator cuff injury following antegrade nailing are of concern and hence other options are often tried [2,3]. The retrograde nail fixation which has gained popularity to avoid this problem needs the patients in prone or lateral decubitus position during surgery.

The authors report that performing Multiple Elastic retrograde intramedullary Nailing in the supine position in adult humeral fractures provides the advantages of both retrograde nailing and supine position during surgery, maintaining the biology and hastening union.

\section{Technique}

The surgery is performed with the patient lying in the supine position under brachial block or a general anesthesia. A radiolucent table is placed next to the operating table where the arm of the patient rests. The limb is draped and a block of linen is placed just beneath the fracture site to aid in the reduction. The arm is abducted to $20^{\circ}$, the elbow is flexed to around $90^{\circ}$ by the assistant. The plan is to get a three point fixation to stabilize the fracture.

The lateral condyle is palpated and the incision is taken. The entry point is marked and checked under the $C$ arm. We prefer to first retract the soft tissues and get an access to the proposed entry point of the lateral condyle just above the lateral supracondylar ridge.

The area is drilled gradually, first with the $2.5 \mathrm{~mm} \mathrm{~K}$ wire perpendicular to the shaft and thereafter with a $3.2 \mathrm{~mm}$ drill bit directed in the cephalad direction within the medullary cavity. The small awl is then directed towards the medullary canal and the hole is gradually enlarged to accommodate atleast two nails from either side. A small elastic titanium nail is negotiated towards the fracture ends.

The limb is then manipulated and the titanium nail is advanced gradually. This acts like an initial reduction nail.
The bent tip of nail from lateral epicondyle, which can be manipulated is intended to end up to the greater tuberosity of Humerus.

Once the nail is beyond the fracture fragments, we approach the medial aspect.

The medial epicondyle is palpated and a small incision is then made over it for the passage of nail after abducting the limb. The incision is extended and care is taken to protect the ulnar nerve by performing the blunt dissection down to the medial epicondyle. The area is drilled gradually, first with the $2.5 \mathrm{~mm} \mathrm{~K}$ wire perpendicular to the shaft and thereafter with a small curved awl directed in the cephalad direction to get an access to the medullary canal. The second titanium nail of adequate length is then selected, is prebent and advanced across the fracture site. It is advanced as close to the proximal humerus to embed into neck of humerus.

Further nails of unequal size are advanced within the fenestration made in the distal cortex to fill the medullary canal and splint it internally. This is ascertained with the C arm. Care is taken that the nails do not irritate the skin or the soft tissue and are accordingly impacted. The skin is closed with 2-0 silk (Figure 1 and Figure 2).

For initial three weeks plaster $U$ slab were given to avoid distraction at the fracture site. Physiotherapy in form of biceps and triceps strengthening exercises, static and dynamic exercises and shoulder pendulum exercises with the humerus braces. The follow up X-ray at nine months showed good consolidation (Figure 3) with no migration of the nails and clinically full function of shoulder and elbow.

*Corresponding author: Yogesh S Salphale, Consultant Trauma Surgeon, Department of Orthopaedics, Shushrusha Multispecialty Hospital, Lane Opp. Z.P, Chandrapur 442401, India, Tel: 00919370323303

Accepted: October 19, 2020

Published online: October 21, 2020

Citation: Salphale YS, Ortho D, Gadegone W (2020) Multiple Elastic Retrograde Intramedullary Nailing for Adult Humeral Diaphyseal Fractures. J Orthop Surg Tech 3(2):191-193 


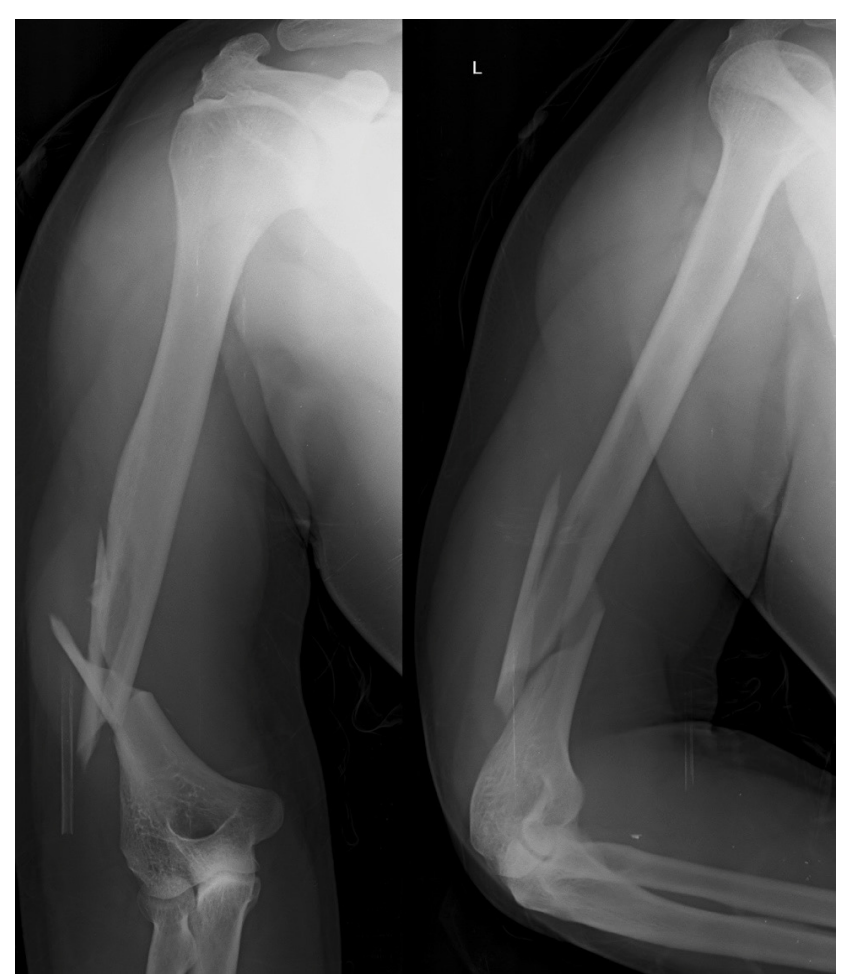

Figure 1: Preoperative image of a young male patient showing the communited fracture of the distal third of the humerus [Holstein-Lewis fracture].

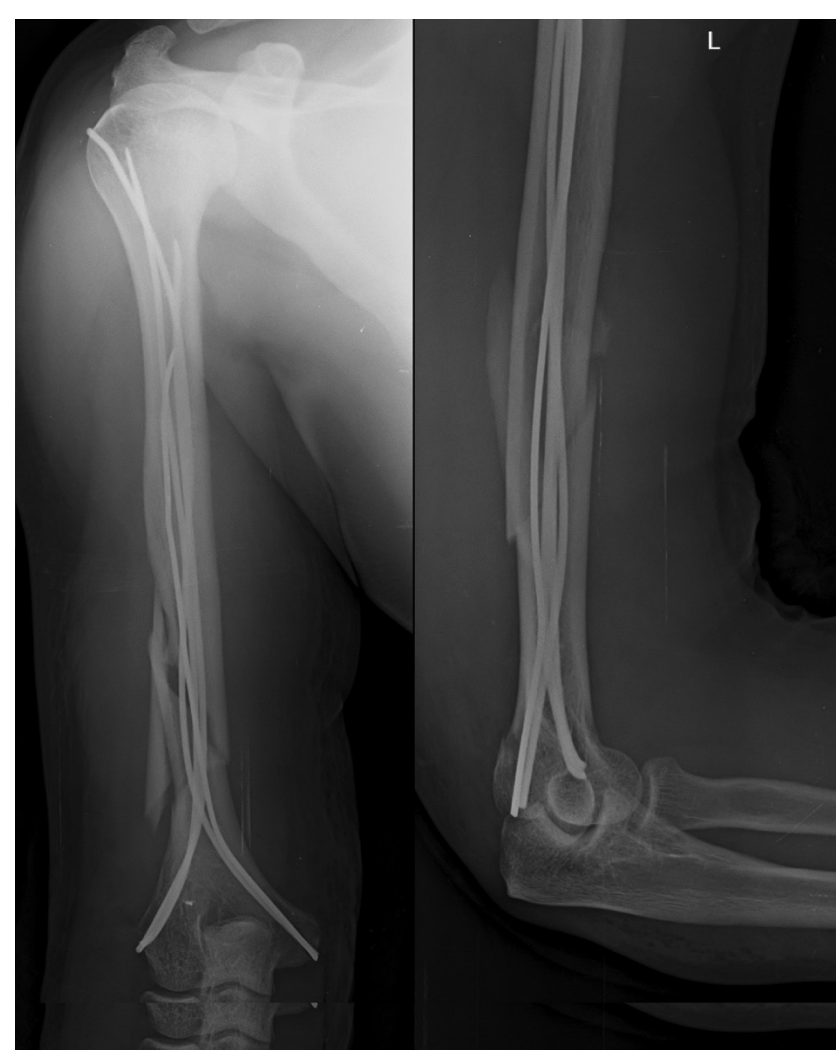

Figure 2: Postoperative image showing the communited fracture fixed with multiple elastic retrograde intramedullary nailing fixation technique.

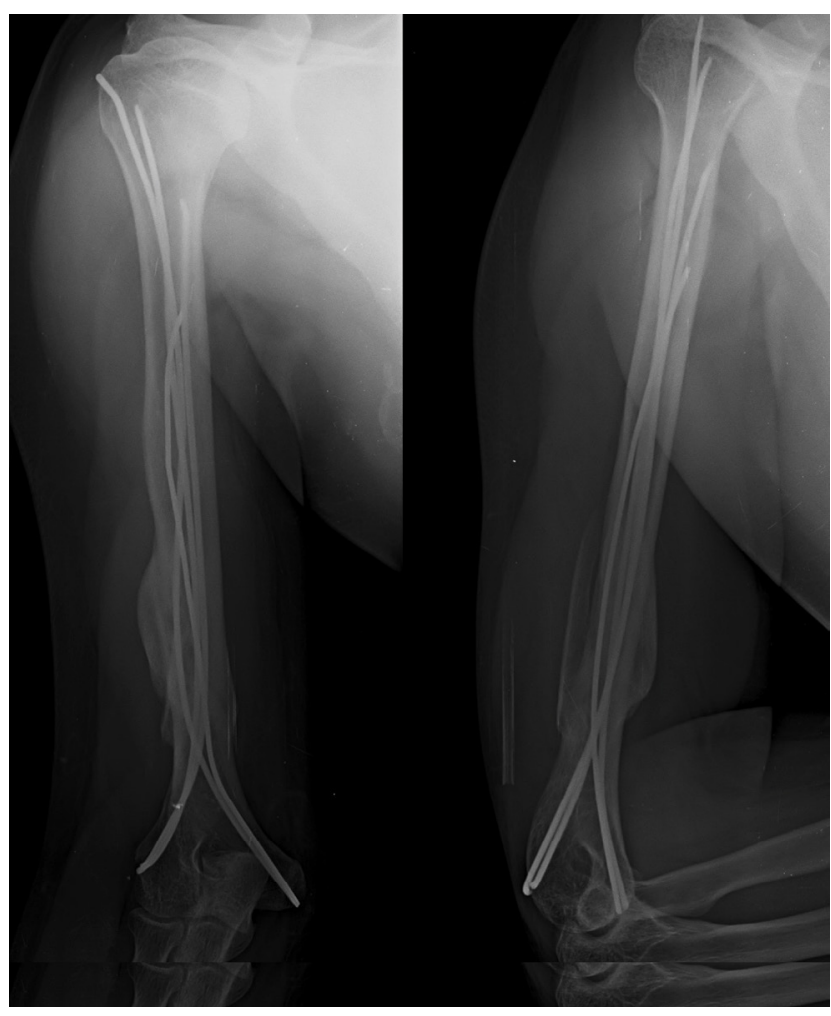

Figure 3: Follow up image at 9 months showing the healed fracture and good callus with multiple elastic retrograde intramedullary nails in situ.

\section{Discussion}

The anatomy and the biomechanical characteristics of the humerus are often neglected while surgically managing these fractures [4]. For a successful outcome following the surgical treatment of humeral fractures consideration of the age of the patient, the medical morbidities, the general health of the patient, the time from fracture to treatment, the adequacy of treatment, and the stability of fixation is essential [2,3].

Functional bracing for the humeral fractures can give good results with "adequate" or "relative" stability $[5,6]$.

Unlike the Enders nailing, which are rigid, these elastic nails are malleable and afford metallurgical advantages. The MRI friendly, titanium nails have a modulus of elasticity quite near to the human bone. As a result titanium nails are easier to negotiate through the bone. As the crowding of the elastic nails take place in the medullary canal the bone tension is increased within the nail which improves the three-point fixation [7].

To answer the issues pertaining to the rotator cuff injury, the retrograde IM nailing in humerus shaft fractures has been practised. It is technically more demanding than antegrade nailing to achieve an optimal fracture configuration and a good fracture stability [8,9]. latrogenic crack or fracture of the distal nail entry site and its propagation is a great concern with retrograde IM nailing [10].

The supine position is beneficial in situations when the patient presents with polytrauma. 
Many surgeons are unfamiliar with performing surgery in the prone or lateral decubitus position. Draping and using the $\mathrm{C}$-arm in such situations raises the operative time and the discomfort of the surgeon.

Multiple TENS afford flexural stability, axial stability, translational stability and rotational stability once placed within the medullary canal. The prebend is more difficult to be retained when multiple nails are passed. The maximal stability by endosteal cortical contact above and below the fracture site can be achieved by the prebend. This internal splinting within the medullary canal retains the length and haematoma and the bony consolidation occurs by relative stability. The surgical time for the proximal and distal locking and the operative radiation is greatly reduced.

For conversion to the open reduction and fixation, if the need arises, the soft tissues are relatively untouched by the index surgery. The elbow flexion and extension movements enhance the healing of the bony union by applying further compression at the fracture site.

\section{Conclusion}

Multiple retrograde intramedullary nailing using titanium elastic nails for the shaft humerus fracture is an alternative and effective surgical modality along with the many techniques described in the literature.

It can be easily performed in any setup with minimal instrumentation. As the soft tissues are relatively untouched, Should the fracture goes into non-union conversion to an open reduction and internal fixation with or without the bone grafting is possible.

We strongly feel that while getting an entry into the medullary canal of the distal humerus, patience and meticulousness is essential. Though Interlocking nailing or biological plating have been described in the literature we feel the advantages of minimal incisional scar, negligible soft tissue damage, low learning curve, economical implants, reduction of operative time, decreased radiation exposure, avoidance of iatrogenic nerve injuryand without affecting shoulder and elbow function are a few points on which this technique scores.

\section{References}

1. Yi JW, Lee JS, Cho HJ (2017) Retrograde intramedullary nailing for humerus fracture in a supine position: Performing an unfamiliar procedure in a familiar position. Clin Orthop Surg 9: 392-395.

2. Gadegone WM, Salphale YS (2008) Antegrade rush pinning for fractures of humeral shaft-A retrospective analysis of 200 cases. European Journal of Orthopaedic Surgery\& Traumatology 18: 93-99.

3. Gadegone WM, Salphale YS (2006) Antegrade rush nailing for fractures of humeral shaft-an analysis of 200 cases with an average follow up of 1 year. Indian Journal of Orthopaedics 40: 180-182.

4. Garnavos C (2011) Diaphyseal humeral fractures and intramedullary nailing: Can we improve outcomes? Indian J Orthop 45: 208-215.

5. Perren SM (2002) Evolution of the internal fixation of long bone fractures. The scientific basis of biological internal fixation: Choosing a new balance between stability and biology. J Bone Jt Surg 84: 1093-1110.

6. Sarmiento A, Zagorski JB, Zych GA, et al. (2000) Functional bracing for the treatment of fractures of humeral diaphysis. J Bone Jt Surg 82: 478-486.

7. Hall RF Jr, Pankovich AM (1987) Ender nailing of acute fractures of the humerus. A study of closed fixation by intramedullary nails without reaming. J Bone Joint Surg Am 69: 558-567.

8. Strohm PC, Reising K, Hammer T, et al. (2011) Humerus shaft fractures: Where are we today? Acta Chir OrthopTraumatol Cech 78: 185-189.

9. Verdano MA, Pellegrini A, Schiavi P, et al. (2013) Humeral shaft fractures treated with antegrade intramedullary nailing: What are the consequences for the rotator cuff? Int Orthop 37: 20012007.

10. Baltov A, Mihail R, Dian E (2014) Complications after interlocking intramedullary nailing of humeral shaft fractures. Injury 45: S9S15.

DOI: $10.36959 / 453 / 545$

Copyright: (c) 2020 Salphale YS, et al. This is an open-access article distributed under the terms of the Creative Commons Attribution License, which permits unrestricted use, distribution, and reproduction in any medium, provided the original author and source are credited. 